\title{
Downregulation of HSPA2 inhibits proliferation via ERK1/2 pathway and endoplasmic reticular stress in lung adenocarcinoma
}

\author{
Longxiang Cao, Xiaoshuai Yuan, Feichao Bao, Wang Lv, Zhehao He, Jie Tang, Jia Han, Jian Hu \\ Department of Thoracic Surgery, The First Affiliated Hospital, School of Medicine, Zhejiang University, Hangzhou 310003, China \\ Contributions: (I) Conception and design: L Cao, X Yuan; (II) Administrative support: W Lv, J Hu; (III) Provision of study materials or patients: W \\ Lv, F Bao; (IV) Collection and assembly of data: F Bao, W Lv, Z He, J Tang; (V) Data analysis and interpretation: L Cao, X Yuan, F Bao, W Lv, Z \\ He; (VI) Manuscript writing: All authors; (VII) Final approval of manuscript: All authors. \\ \#These authors contributed equally to this work. \\ Correspondence to: Jian Hu. Department of Thoracic Surgery, The First Affiliated Hospital, School of Medicine, Zhejiang University, Hangzhou \\ 310003, China. Email: dr_hujian@zju.edu.cn.
}

\begin{abstract}
Background: To explore the mechanisms of HSPA2 downregulation in inhibiting the proliferation of lung adenocarcinoma.

Methods: We obtained 85 specimens of human lung adenocarcinoma and specimens of adjacent nontumor tissues from the First Affiliated Hospital, School of Medicine, Zhejiang University. We then analyzed the expression of HSPA2 in these tissues and in lung adenocarcinoma and normal lung cell lines. Human lung adenocarcinoma cell lines were transfected with siRNA silencing HSPA2 and subjected to colony forming, Thiazolyl blue tetrazolium bromide (MTT), propidium iodide flow cytometry, immunofluorescence assay and western blotting to explore the causes of the reduction in the proliferation of lung adenocarcinoma cells and the endoplasmic reticulum stress induced by HSPA2 downregulation. Finally, we confirmed these mechanisms via rescue assay.

Results: Greater HSPA2 expression was found in the lung adenocarcinoma specimens than in the specimens of adjacent nontumor tissues, and greater expression was found in lung adenocarcinoma cell lines than in normal cell lines. HSPA2 knockdown via siRNA reduced proliferation and led to G1/S phase cell cycle arrest in the lung adenocarcinoma cell lines. G1/S phase cell cycle arrest triggered by HSPA2 downregulation could be attributed, at least in part, to phosphorylation and activation of the Erk1/2 pathway and probably to activation of IRE1 $\alpha /$ PERK-mediated endoplasmic reticulum stress.
\end{abstract}

Conclusions: HSPA2 plays an important role in the origin and development of lung adenocarcinoma. It is thus deserving of further study as a promising clinical therapeutic target.

Keywords: Lung adenocarcinoma; HSPA2; G1/S cell cycle arrest; Erk1/2

Submitted Sep 11, 2019. Accepted for publication Sep 25, 2019.

doi: $10.21037 /$ atm.2019.10.16

View this article at: http://dx.doi.org/10.21037/atm.2019.10.16

\section{Introduction}

Malignant tumors are among the most horrible diseases of the 21 st century, and lung cancer is the most commonly diagnosed cancer and the leading cause of cancer death (1). Most lung cancer diagnoses (85\%) are non-small cell lung cancer (NSCLC) (2), including lung adenocarcinoma. The 5 -year survival estimates for NSCLC range from $73 \%$ for stage IA disease to $13 \%$ for stage IV disease (3). The increasing morbidity rates of lung adenocarcinoma has led to a relative flood of research.

HSPA2 is a variant in the HSP70 family and it is an essential in spermiogenesis. In oncology research, HSPA2 was first reported in renal cell carcinoma (4). Several studies have shown that HSPA2 plays important roles in the cell growth and proliferation of hepatocellular carcinoma, 
nasopharyngeal carcinoma, esophageal squamous cell carcinoma, NSCLC, pancreatic ductal adenocarcinoma, colorectal carcinoma and breast carcinoma (5-10). Increased expression of $H S P A 2$ was significantly related to a shorter overall duration of survival in patients with stage I-II carcinoma (7).

However, unlike other members of the HSP70 family, the function of HSPA2 in carcinogenesis and the development of lung cancer has rarely been reported. We thus explored the role of $H S P A 2$ in lung cancer cell proliferation to determine how the downregulation of HSPA2 inhibits the proliferation of lung adenocarcinoma cells via multiple mechanisms.

\section{Methods}

\section{Patients and tissue specimens}

Eight-five clinical pathology specimens of lung adenocarcinoma tissue and matched adjacent nontumor tissue were collected from the Department of Thoracic Surgery, the First Affiliated Hospital, School of Medicine, Zhejiang University with the approval of Research Ethics Committee of the First Affiliated Hospital, College of medicine, Zhejiang University. The procedure was conducted in accordance with the 1964 Declaration of Helsinki and its later amendments or comparable ethical standards. All patients had given signed informed consent. The specimens of adjacent nontumor tissue for each patient were obtained more than $3 \mathrm{~cm}$ from carcinoma tissue.

\section{Cell lines and cell culture}

We used human lung adenocarcinoma cell lines (including A549, H1975, and H292), human bronchial epithelium, and human embryonic lung fibroblasts (MRC-5) for our experiments. All cell lines were acquired from the Cell Bank of the Chinese Academy of Sciences (Shanghai, China). They were cultured at $37^{\circ} \mathrm{C}$ in a humidified atmosphere (Thermo Fisher Scientific) in the presence of $5 \% \mathrm{CO}_{2}-$ 95\% air and were grown in RMPI-1640 supplemented with $10 \%$ Fetal Bovine Serum (FBS).

\section{Real-time PCR}

Total RNA was extracted from tissues by TRIzol reagent (Invitrogen Life Technologies) and used to synthesize cDNA (Bio-Rad, Hercules, CA, USA). Real-time PCR was performed according to standard protocols.

\section{Transfection of HSPA2 siRNA and overexpressing plasmid}

The cells were plated in six-well plates and allowed to attach overnight. With the application of lipofectamin2000, HSPA2 siRNA sequenced and overexpressing plasmid was transfected into A549 and H1975 cell lines to knockdown or overexpress the HSPA2 gene respectively.

\section{Colony formation assay and MTT assay}

After the cells being transfected as needed, colony formation assay was performed in six-well plates with cell culture for 14 days without disturbance. We washed the media in triplicate with phosphate-buffered saline (PBS) solution followed by $0.1 \%$ crystal violet for 15 minutes.

We seeded A549 and H1975 cells (being transfected as needed) into 96 -well plates at $80 \%$ to $90 \%$ subconfluence. MTT was added to the medium for 3 hours of incubation after 24,48 , and 72 hours of incubation. The cell culture medium was then mixed with $150 \mu \mathrm{L}$ of dimethyl sulfoxide for 10 minutes, and the optical absorbance was measured at $490 \mathrm{~nm}$.

\section{Immunofluorescence assay}

We performed transfection in culture dishes and labeled ER with ER-Tracker ${ }^{\text {TM }}$ Green dyes (Invitrogen Life Technologies) according to the instructions of the manufacturer. Washed the cell for three times with PBS for 5 minutes and visualized at room temperature using a Carl Zeiss microscope (Carl Zeiss AG, Jena, Germany). The exposure times between treatments were consistent and the brightness and contrast of the images were adjusted using Adobe Photoshop software (Adobe Systems Inc., San Jose, CA, USA) for presentation.

\section{Cell cycle assay}

We performed flow cytometry according to standard protocols of DNA staining solution (Multisciences Biotech Co., Ltd., China). After transfection, the cells were collected and washed with PBS. The cell pellets were obtained by centrifugation and the supernatant was discarded. Then added $1 \mathrm{~mL}$ of DNA staining solution incubated the cell samples for 30 minutes at room temperature in the dark for the flow cytometry. 

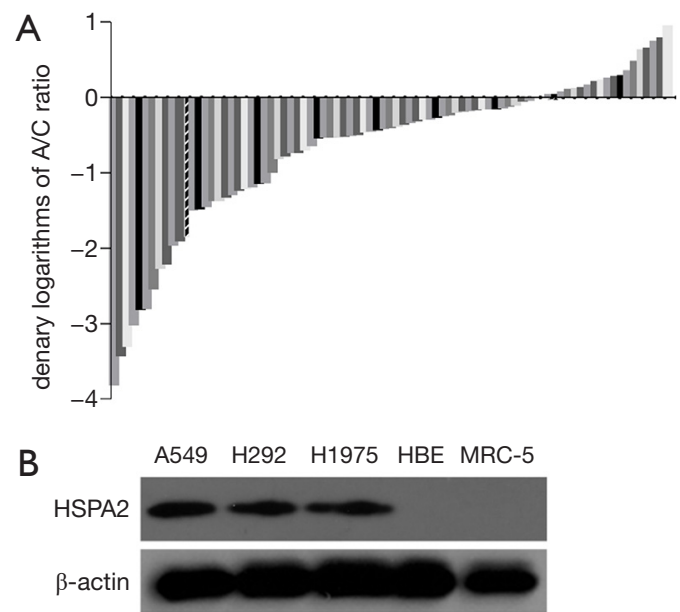

Figure 1 Expression of HSPA2 was higher in tissue and cell lines of lung adenocarcinoma. (A) Expression level comparison of HSPA2 in lung adenocarcinoma specimens and specimens of adjacent nontumor tissue. HSPA2 expression was greater in the lung adenocarcinoma specimens than in those of adjacent nontumor tissue. The denary logarithms of the $\mathrm{A} / \mathrm{C}$ ratio (C indicates the expression of HSPA2 in cancer tissue, and $\mathrm{A}$ indicates the expression of HSPA2 in the adjacent nontumor tissue, all with $\triangle \mathrm{CT}$ value) are shown from low to high. Negatives showed greater expression in lung adenocarcinoma specimens than in those of adjacent nontumor tissues. (B) Expression level comparison of HSPA2 in lung adenocarcinoma and normal cell lines. Western blot result shows greater HSPA2 expression in lung cancer cell lines (A549, H1975, H292) than in normal lung cell lines (human bronchial epithelium, MRC-5).

\section{Western blot}

The cells were sonicated in the proper amount of radioimmunoprecipitation assay buffer, and $20 \mu \mathrm{g}$ of protein was resolved on $12 \%$ polyacrylamide sodium dodecyl sulfate gels. The proteins were then transferred to polyvinylidene difluoride membranes, which were blocked with $3 \%$ bovine serum albumin in Tris-buffered saline-Tween 20 . The membranes were incubated successively with the corresponding primary antibodies and secondary antibodies. Finally, the proteins were visualized with chemiluminescent horseradish peroxidase substrate.

\section{Statistical analysis}

All experiments were performed in triplicate, and the results were analyzed with GraphPad Prism 7.0. P values of less than 0.05 were considered to indicate statistical significance.

\section{Results}

HSPA2 showed greater expression in tissue and cell lines of lung adenocarcinoma than in adjacent nontumor tissue and normal cells

We applied qRT-PCR to measure tissue specimens from 85 patients. Every column represented one patient, and the $\mathrm{A} / \mathrm{C}$ ratio indicated the ratio of PCR results between group A (adjacent nontumor tissues) and group C (lung adenocarcinoma tissues). The qRT-PCR results of demonstrated that HSPA2 showed greater expression in lung adenocarcinoma tissues than in the adjacent nontumor tissues (Figure 1A).

Western blotting showed that the lung adenocarcinoma cell lines had a significantly higher expression level of HSPA2 than the human bronchial epithelium and MRC-5 cell lines (Figure 1B).

\section{Knockdown of HSPA2 reduced proliferation of adenocarcinoma cells}

Selection of an effective siRNA sequence to knock down HSPA2 gene expression

We found siRNA sequence no.1532 to be the most effective, so it was applied in the subsequent experiments (Figure $2 A$ ).

\section{Knockdown of HSPA2 reduced proliferation of A549 and $\mathrm{H} 1975$ cells}

MTT assay and colony formation assay demonstrated that knockdown of HSPA2 reduced the proliferation of A549 and H1975 cells (Figure 2B,C).

\section{Knockdown of HSPA2 induced G1/S phase cell cycle arrest of $A 549$ and $\mathrm{H} 1975$ cells}

The results of flow cytometry show that G1 phase cell increased and G2 phase cell decreased in the HSPA2 knockdown group of A549 and H1975 cell lines (Figure 3A). These results indicated that knockdown of HSPA2 induced G1/S phase cell cycle arrest.

Cell cycle regulation proteins, including CDK4, cyclin D1, cyclin D3, and cyclin E1, showed reduced expression in the HSPA2 knockdown group. G1/S phase checkpoint protein $\mathrm{p}-\mathrm{Rb}(\mathrm{S} 795)$ and $\mathrm{p}-\mathrm{Rb}(\mathrm{S} 807 / 811)$ were also downregulated, whereas $\mathrm{Rb}$ protein showed no significant difference between the two groups (Figure 3B). 

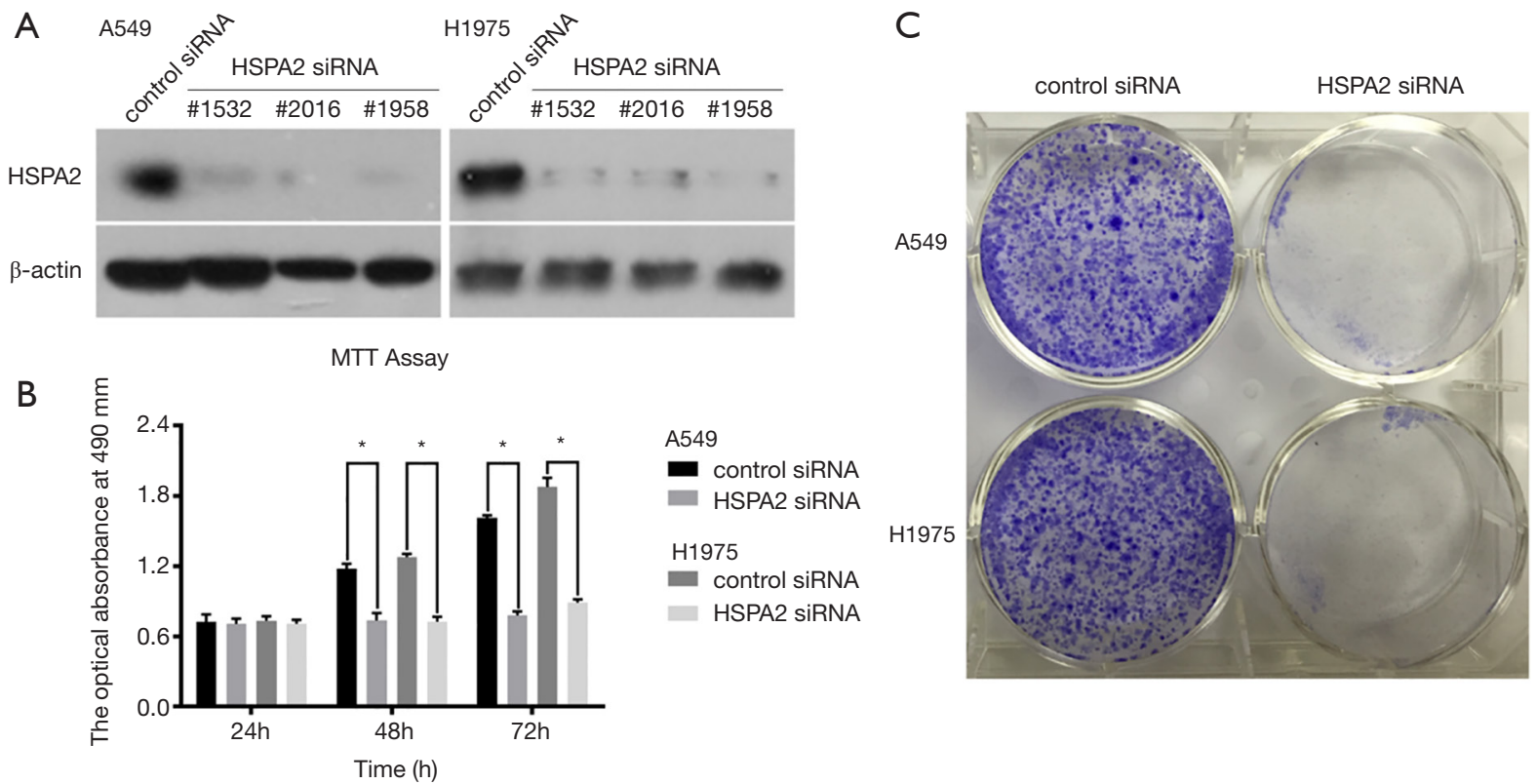

Figure 2 Knockdown of HSPA2 reduced proliferation of adenocarcinoma cells. (A) Effects of HSPA2 siRNA on the expression of HSPA2. Western blot analysis demonstrates the effect of HSPA2 siRNA transfection on HSPA2 expression in A549 and H1975 cells. (B) HSPA2 knockdown reduced the proliferation of A549 and H1975 cells by MTT assay. The values obtained from the transfected and control cells represent the mean \pm SD of three independent experiments. (C) HSPA2 knockdown reduced the proliferation of A549 and H1975 cells by representative colony formation assay. ${ }^{*} \mathrm{P}<0.05$.

\section{Knockdown of HSPA2 activated Erk1/2 signaling transduction patbway and Erk1/2 inbibitor rescue experiment}

We found an increased phosphorylation level of Erk1/2 protein in both A549 and H1975 cell lines. The total protein level, relative protein level of STAT3, and the Akt pathway remained stable (Figure $4 A$ ). We presumed that HSPA2 could participate in the Erk1/2 cell signal transduction pathway. Activation of the Erk1/2 pathway induces cell cycle arrest in lung adenocarcinoma.

To confirm the former result, we rescued the G1/S phase cell cycle arrest by 24-hour treatment with Selumetinib (specific phosphorylation Erk1/2 inhibitor) after HSPA2 knockdown in A549 and H1975. Figure 4B shows that Selumetinib was able to rescue cell cycle arrest of A549 and H1975 cells.

\section{Knockdown of HSPA2 enbanced ER stress in A549 and H1975 cells}

\section{Immunofluorescence}

We hypothesized that organelles may participate in the process of G1/S phase arrest induced by HSPA2 knockdown.
We thus selected a specialized endoplasmic reticulum (ER) marker to stain the ER of A549 and H1975 transfected by HSPA2 siRNA or blank siRNA. We then observed them via laser confocal microscopy (LCM). Immunofluorescence demonstrated greater signal enhancement in the HSPA2 knockdown group than in the control group (Figure $5 A$ ), which indicates an increase in ER stress (11). These results confirmed our hypothesis.

\section{Western blot}

We focused the ER stress signaling pathway protein (ATF6, IRE1 $\alpha$, PERK) and Bip (modulin of IRE1 $\alpha$ protein). Western blotting showed that an increase in the expression levels of IRE1 $\alpha$ and PERK and a decrease in Bip (Figure 5B).

\section{Rescue assay via HSPA2 knockdown and overexpression}

We observed the expression levels of HSPA2, CDK4, cyclin $\mathrm{D} 1$, cyclin $\mathrm{D} 3$, cyclin $\mathrm{E} 1, \mathrm{p}-\mathrm{Rb}(\mathrm{S} 795), \mathrm{p}-\mathrm{Rb}(\mathrm{S} 807 / 811)$, p-Erk1/2, IRE1 $\alpha$, PERK, and Bip in the previous experiments. HSPA2 overexpressing plasmid was applied in A549 cell line. By comparing the changing protein levels in the control group (Con), the knockdown group (KD), the overexpression group (OE), and the rescue group (KD + 


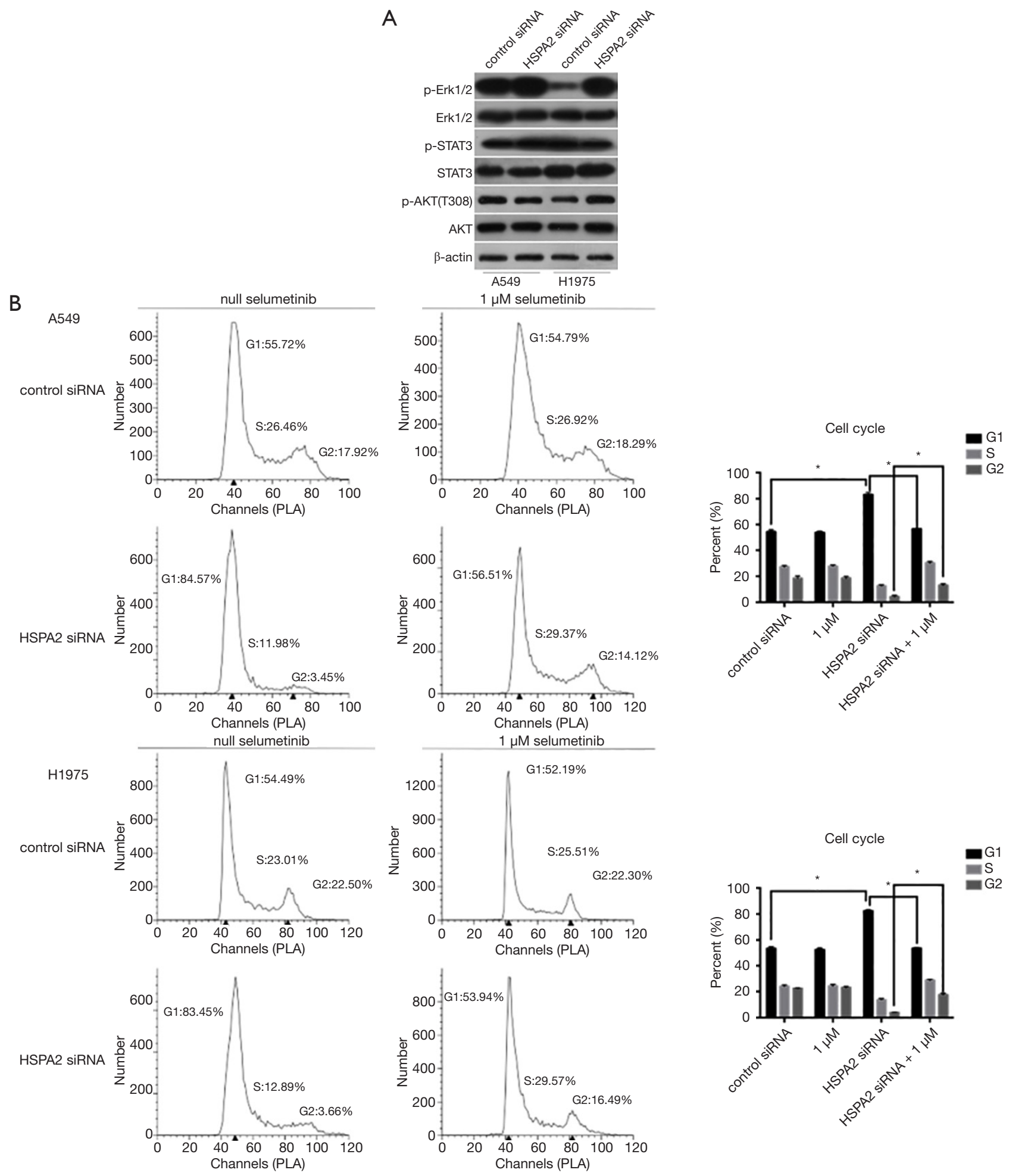

Figure 3 Knockdown of HSPA2 induced G1/S phase cell cycle arrest. (A) HSPA2 knockdown induced the G1/S phase cell cycle arrest of A549 and H1975 cells. Cell cycle profiles of A549 and H1975 cells transfected with HSPA2 siRNA and control cells were determined by flow cytometry and error bars. Cell count versus PI staining is shown (10,000 per treatment). (B) HSPA2 knockdown induced the change of G1/S phase regulation and checkpoint proteins of A549 and H1975 cells. Western blot analysis of G1/S phase cell cycle regulation and checkpoint proteins with A549 and H1975 cell transfected with HSPA2 siRNA and control cells are shown. *, $\mathrm{P}<0.05$. 

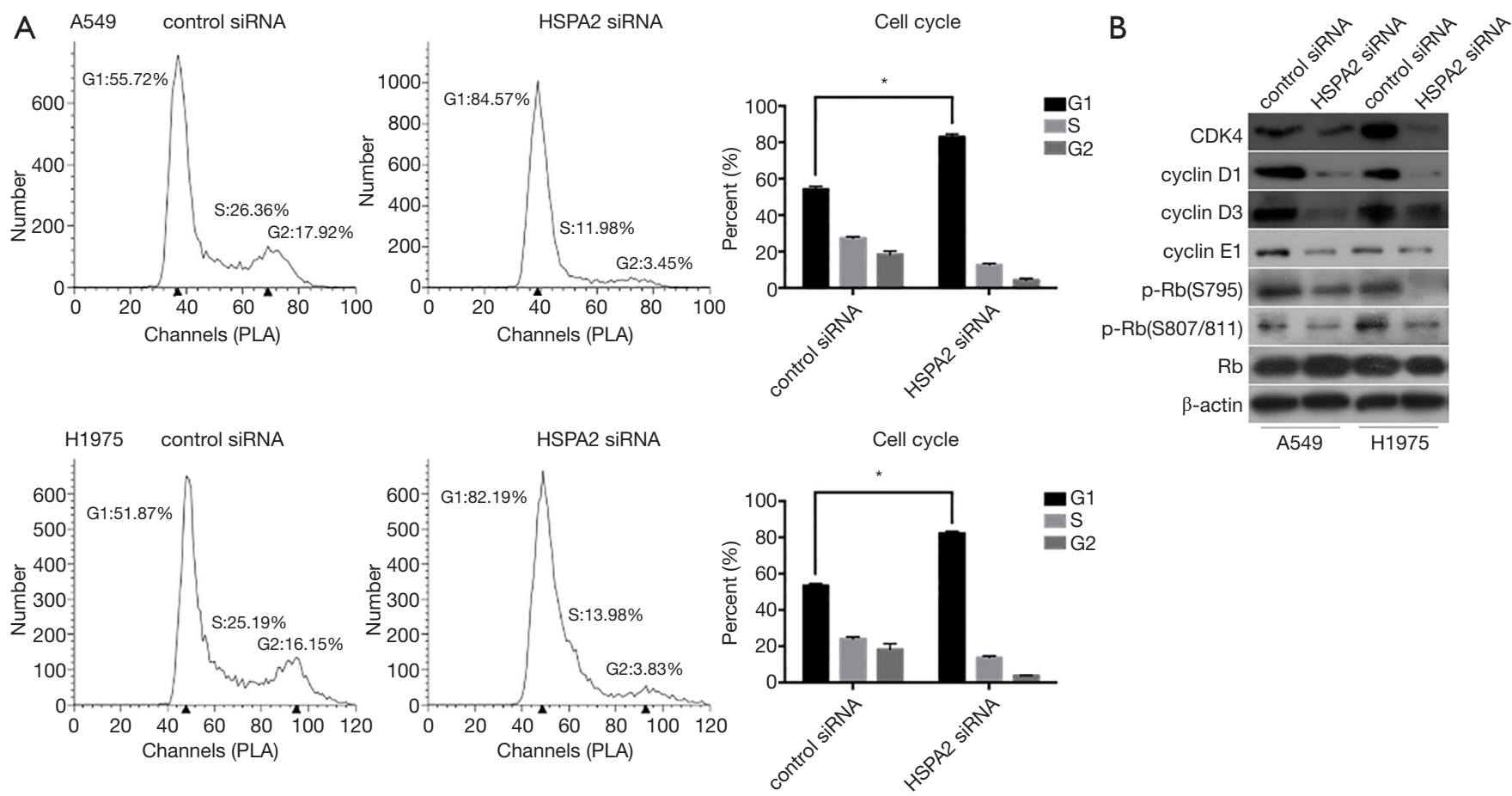

Figure 4 Knockdown of HSPA2 activated Erk1/2 signaling transduction pathway. (A) Downregulation of HSPA2 related with Erk1/2 signaling transduction pathway and c-Myc protein. Western blot analysis of proteins in pathways involved in cell cycle regulation with A549 and H1975 cells transfected with HSPA2 siRNA and control cells. (B) Verification of the finding that HSPA2 knockdown triggers the activation of the Erk1/2 pathway. Cell cycle profiles of A549 and H1975 cells with or without HSPA2 knockdown or Selumetinib (1 $\mu$ M) and error bars were determined by flow cytometry. *, $\mathrm{P}<0.05$.

$\mathrm{OE})$, we confirmed the conclusion that downregulation of $H S P A 2$ inhibits proliferation via the ERK1/2 pathway and ER stress in lung adenocarcinoma (Figure 6).

\section{Discussion}

Ferrucio Ritossa proposed the existence of the heat shock response in the 1960s (12). It is now recognized that the heat shock response is widespread in most kinds of species and that cells surrounding the exchanging protein structure would show a heat shock response. A major characteristic of the heat shock response is that it encodes the gene signals of heat shock protein (HSPs) and then induces and amplifies the follow-up biological response. Heat shock response decreases the negative effect by means of environmental or endogenous molecular stress (13).

Molecular chaperone HSPs control protein folding to participate in the intracellular balance. Some HSPs serve as the molecular chaperones of other hyperspecialization proteins, such as signal transduction proteins and transcription factors.
Some HSPs show resistance to apoptosis and participate in some cellular immune responses (14). These findings have attracted scholars to study and induced lots of clinical applications.

The largest family of HSPs is the HSPA (HSP70) family, which belongs to the human protein group (15). HSPA2 is a variant of this family. It was first found in testicular tissue and is highly expressed in pachytene stage spermatocytes in rodents. We therefore regard HSPA2 as a specific expression protein of the testes (16).

HSPA2 protein is thought to be abnormally expressed in lung alveolar carcinoma (17). The construction of HSPA2 protein directly influences its regulated function in the progression of lung cancer (18). Biomarkers $(7,19,20)$ and polymorphism (21) analysis have been the main achievements of HSPA2 research for lung cancer, but studies of the mechanism are rare. In 2016, Chang et al. showed interaction between HSPA2 and JAG1, one of the ligands in the Notch pathway. The improved transcriptional level of JAG1 was related with HSPA2, and both participated in the 


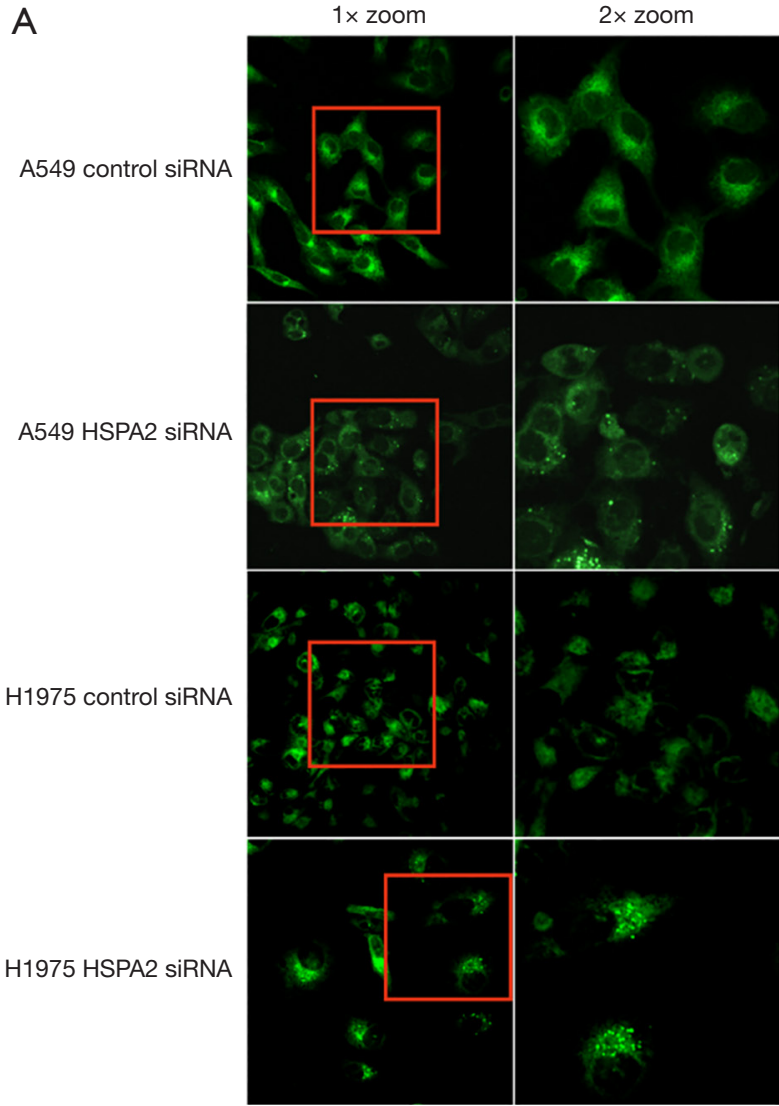

B
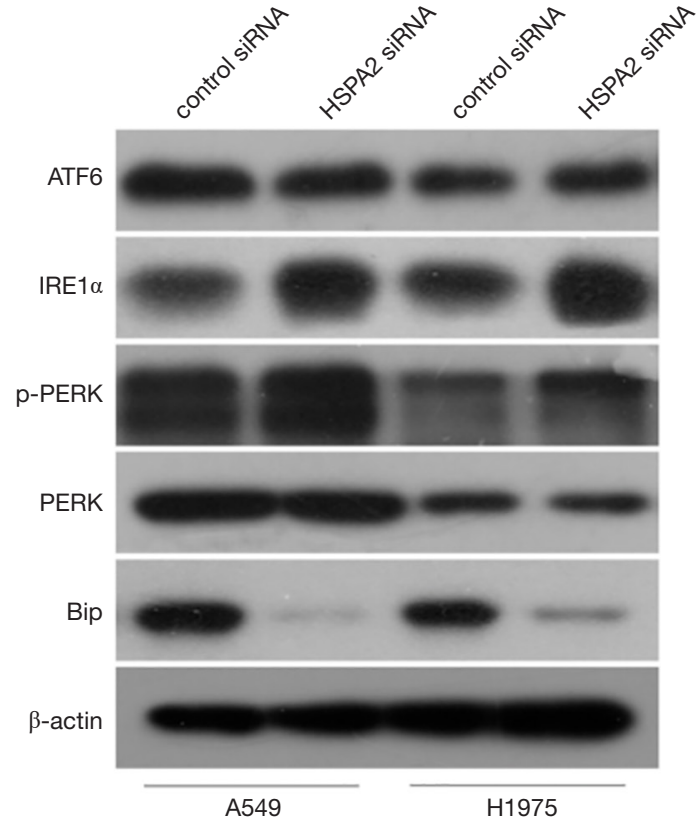

Figure 5 Knockdown of HSPA2 enhanced ER stress. (A) HSPA2 participates in the ER stress of A549 and H1975 cells. Representative ERTracker green dye assays of HSPA2 siRNA-transfected and control A549 and H1975 cells. Highlights of the cells represent the occurrence of ER stress. Left: 1×Zoom; Right: $2 \times$ Zoom. (B) Verification of the participation of HSPA2 in the ER stress of A549 and H1975 cells. Western blot analysis of proteins in ER stress pathways with A549 and H1975 cells transfected with HSPA2 siRNA and control cells.
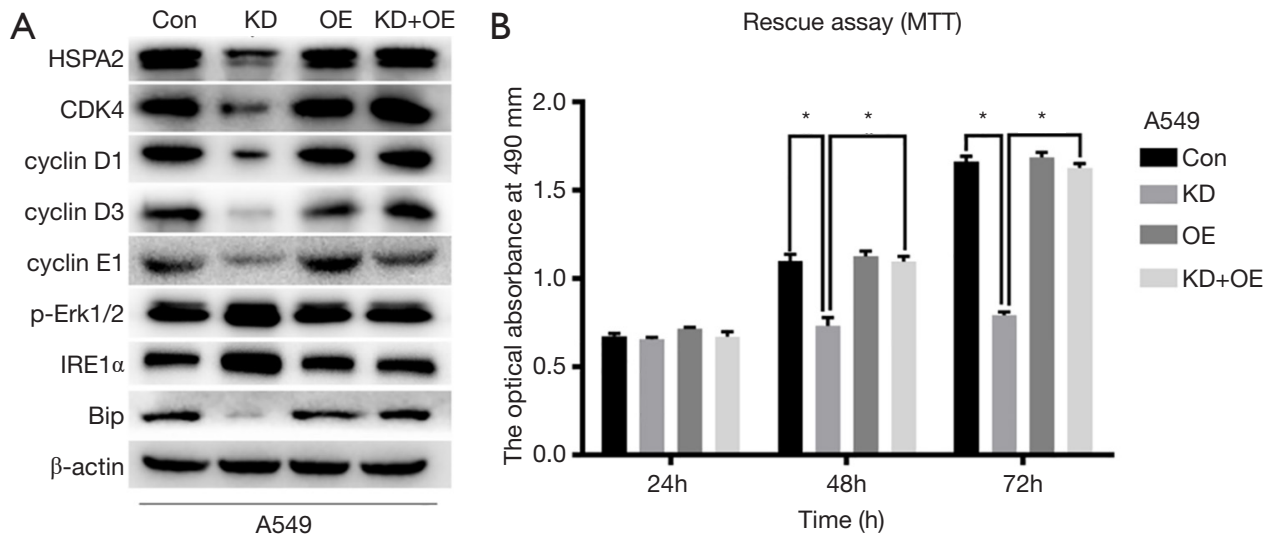

Figure 6 Rescue assay via HSPA2 knockdown and overexpression. (A) Rescue assay shows that HSPA2 overexpression and plasmid transfection could rescue the downregulation of HSPA (HSP70), CDK4, cyclin D1, cyclin D3, cyclin E1 and Bip. Meanwhile, the overexpression of p-Erk1/2 and IRE1 $\alpha$ were reversed. (B) HSPA2 knockdown reduced the proliferation of A549 cells and HSPA2 overexpression reversed the decreasing trend by MTT assay. The values obtained from the transfected and control cells represent the mean \pm SD of three independent experiments. *, $\mathrm{P}<0.05$. 
process of malignant transformation of lung cancer (22). We have found no further studies of the mechanism of HSPA2 function in lung adenocarcinoma.

In this study, we observed HSPA2 expression in lung adenocarcinoma tissue and relative cell lines. Our results show greater HSPA2 expression in the lung adenocarcinoma specimens than in the adjacent nontumor tissues, which was confirmed by western blot of the cell lines.

The proliferation of lung adenocarcinoma cell lines (A549 and H1975) decreased after HSPA2 knockdown, which was induced by G1/S phase cell cycle arrest as the expression of relative protein in the G1/S phase cell cycle changed accordingly. After downregulation of $H S P A 2$, western blotting showed increasing phosphorylation of Erk1/2. The extracellular signal-regulated kinase ERK1 and ERK2 (ERK1/2) cascade regulates a variety of cellular processes via phosphorylation of multiple target proteins. The outcomes of its activation range from stimulation of cell survival and proliferation to triggering of tumor suppressor responses such as cell differentiation, cell senescence, and apoptosis (23). The hyperphosphorylation of Erk1/2 protein could promote cell cycle arrest by regulating p16 (24), p21 (25), and p27 (26), which are common inhibitors of G1/S cyclin-dependent kinase. The function of increasing the phosphorylation level of Erk1/2 protein in cell cycle regulation was consistent with our results.

The ER is the major site of protein synthesis and transport in eukaryotic cells and can transport abnormal proteins to the cytoplasm. Those proteins were degraded by proteasome via ER-associated degradation. A disorder of ER-associated degradation would induce ER stress and trigger the unfolded protein response. IRE1 $\alpha$, PERK, and ATF6 protein represent three branches of the unfolded protein response (27). Studies of ER stress in the regulation of tumor cell cycle arrest have been reported $(28,29)$. Meanwhile, the functions of HSPs in ER stress have been frequently studied (30-34). However, the relationship between HSPA2 and ER stress remains unknown. Our study shows that upregulation level of IRE1 $\alpha$ and PERK protein in the HSPA2 knockdown group is comparable with that in the control group. It is worth noting that the Erk1/2 cascade have a reported relationship with ER stress. Erk1/2 has been intimately linked with the IRE $1 \alpha$ and PERK stress pathway. Erk1/2 protein activation was inhibited after ER stress and IRE1 $\alpha$ pathway activation, but the mechanism was unclear (35). A similar phenomenon has been observed in gastric cancer cells, in which activation of Erk1/2 inhibited Bip protein in the IRE1 $\alpha$ pathway, after which apoptosis was induced by ER stress (36).

The activation of Erk1/2 mediated phosphorylation of CHOP (downstream of the PERK pathway). This process could change the activity of its own transcriptional activation domain, subsequently changing its affinity with the binding protein and ultimately leading to changes in the gene expression profiles (37). In a study of HeLa cells, Erk1/2 also played an important role in the process of apoptosis induced by the high expression level of CHOP, and Erk1/2 also mediated the phosphorylation of CHOP (38). It remains uncertain which genes were dependent on $\mathrm{CHOP}$ phosphorylation and were responsible for alteration of the cellular phenotype associated with triggering the subsequent progression of cell apoptosis.

Therefore, when the downregulation of HSPA2 induces ER stress, crosstalk may occur with the high phosphorylation level of Erk1/2. Erk1/2 activation leading to ER stress or ER stress happening before Erk1/2 protein phosphorylation require further study, and the relationship between ER stress and the cell cycle requires further discussion.

\section{Conclusions}

We observed greater expression of HSPA2 in lung adenocarcinoma tumor tissues and cell lines than in the adjacent nontumor tissues and normal cell lines. It was confirmed that HSPA2 gene silencing effectively inhibited the proliferation of lung adenocarcinoma cell lines. HSPA2 gene silencing led to the G1/S phase arrest of A549 and H1975 cells. It was demonstrated that HSPA2 caused G1/ S phase arrest in A549 and H1975 cells via regulation of the Erk1/2 pathway and by triggering ER stress, thus inhibiting the proliferation of A549 and H1975 cells. We confirmed, for the first time, that HSPA2 plays an important role in the occurrence and development of lung adenocarcinoma. It is thus worthy of further study as a promising clinical therapeutic target.

\section{Acknowledgments}

Funding: This study was supported by the National Natural Science Foundation of China (Project 31170720 and Project 81900099), the Traditional Chinese Medical Science and Technology Key Research Plan of Zhejiang Province (Project 2015ZZ007), the Foundation of Zhejiang Provincial Traditional Chinese Medicine Scientific Research Plan (Project 2016ZA125) and "The 13th Five 
Year" Traditional Chinese Medicine (integrated Chinese traditional and Western medicine) Key Discipline of Zhejiang Province (Innovative lung cancer diagnosis and treatment with combination of Chinese traditional and Western medicine 2017-XK-A33).

\section{Footnote}

Conflicts of Interest: The authors have no conflicts of interest to declare.

Ethical Statement: This study has been approved by the ethics committee of the First Affiliated Hospital of Zhejiang University in China and written informed consent was obtained from eligible guardians. The authors are accountable for all aspects of the work in ensuring that questions related to the accuracy or integrity of any part of the work are appropriately investigated and resolved.

\section{References}

1. Bray F, Ferlay J, Soerjomataram I, et al. Global cancer statistics 2018: GLOBOCAN estimates of incidence and mortality worldwide for 36 cancers in 185 countries. CA Cancer J Clin 2018;68:394-424.

2. Brawley OW. Avoidable cancer deaths globally. CA Cancer J Clin 2011;61:67-8.

3. Woodard GA, Jones KD, Jablons DM. Lung Cancer Staging and Prognosis. Cancer Treat Res 2016;170:47-75.

4. Gaudin C, Kremer F, Angevin E, et al. A hsp70-2 mutation recognized by CTL on a human renal cell carcinoma. J Immunol 1999;162:1730-8.

5. Jalbout M, Bouaouina N, Gargouri J, et al. Polymorphism of the stress protein HSP70-2 gene is associated with the susceptibility to the nasopharyngeal carcinoma. Cancer Lett 2003;193:75-81.

6. Zhang H, Chen W, Duan CJ, et al. Overexpression of HSPA2 is correlated with poor prognosis in esophageal squamous cell carcinoma. World J Surg Oncol 2013;11:141.

7. Scieglinska D, Gogler-Piglowska A, Butkiewicz D, et al. HSPA2 is expressed in human tumors and correlates with clinical features in non-small cell lung carcinoma patients. Anticancer Res 2014;34:2833-40.

8. Zhang H, Gao H, Liu C, et al. Expression and clinical significance of HSPA2 in pancreatic ductal adenocarcinoma. Diagn Pathol 2015;10:13.

9. Jagadish N, Parashar D, Gupta N, et al. Heat shock protein 70-2 (HSP70-2) is a novel therapeutic target for colorectal cancer and is associated with tumor growth. BMC Cancer 2016;16:561.

10. Jagadish N, Agarwal S, Gupta N, et al. Heat shock protein 70-2 (HSP70-2) overexpression in breast cancer. J Exp Clin Cancer Res 2016;35:150.

11. Zunkler BJ, Wos-Maganga M, Panten U. Fluorescence microscopy studies with a fluorescent glibenclamide derivative, a high-affinity blocker of pancreatic betacell ATP-sensitive K+ currents. Biochem Pharmacol 2004;67:1437-44.

12. Scieglinska D, Krawczyk Z. Expression, function, and regulation of the testis-enriched heat shock HSPA2 gene in rodents and humans. Cell Stress Chaperones 2015;20:221-35.

13. Gidalevitz T, Prahlad V, Morimoto RI. The stress of protein misfolding: from single cells to multicellular organisms. Cold Spring Harb Perspect Biol 2011;3. doi: 10.1101/cshperspect.a009704.

14. Kakkar V, Meister-Broekema M, Minoia M, et al. Barcoding heat shock proteins to human diseases: looking beyond the heat shock response. Dis Model Mech 2014;7:421-34.

15. Kampinga HH, Hageman J, Vos MJ, et al. Guidelines for the nomenclature of the human heat shock proteins. Cell Stress Chaperones 2009;14:105-11.

16. Roux AF, Nguyen VT, Squire JA, et al. A heat shock gene at 14q22: mapping and expression. Hum Mol Genet 1994;3:1819-22.

17. Fijneman RJ, Oomen LC, Snoek M, et al. A susceptibility gene for alveolar lung tumors in the mouse maps between Hsp70.3 and G7 within the H2 complex. Immunogenetics 1995;41:106-9.

18. Piglowski W, Nowak R, Krawczyk Z, et al. The structural and functional analysis of the human HSPA2 gene promoter region. Acta Biochim Pol 2007;54:99-106.

19. Scieglinska D, Piglowski W, Mazurek A, et al. The HspA2 protein localizes in nucleoli and centrosomes of heat shocked cancer cells. J Cell Biochem 2008;104:2193-206.

20. Scieglinska D, Piglowski W, Chekan M, et al. Differential expression of HSPA1 and HSPA2 proteins in human tissues; tissue microarray-based immunohistochemical study. Histochem Cell Biol 2011;135:337-50.

21. Wang Y, Zhou F, Wu Y, et al. The relationship between three heat shock protein 70 gene polymorphisms and susceptibility to lung cancer. Clin Chem Lab Med 2010;48:1657-63.

22. Chang WH, Ho BC, Hsiao YJ, et al. JAG1 Is Associated 
with Poor Survival through Inducing Metastasis in Lung Cancer. PLoS One 2016;11:e0150355.

23. Deschenes-Simard X, Kottakis F, Meloche S, et al. ERKs in cancer: friends or foes? Cancer Res 2014;74:412-9.

24. Kim JY, Choi JA, Kim TH, et al. Involvement of p38 mitogen-activated protein kinase in the cell growth inhibition by sodium arsenite. J Cell Physiol 2002;190:29-37.

25. Faust D, Dolado I, Cuadrado A, et al. p38alpha MAPK is required for contact inhibition. Oncogene 2005;24:7941-5.

26. Kwong J, Hong L, Liao R, et al. p38alpha and p38gamma mediate oncogenic ras-induced senescence through differential mechanisms. J Biol Chem 2009;284:11237-46.

27. Walter P, Ron D. The unfolded protein response: from stress pathway to homeostatic regulation. Science 2011;334:1081-6.

28. Bourougaa K, Naski N, Boularan C, et al. Endoplasmic reticulum stress induces $\mathrm{G} 2$ cell-cycle arrest via mRNA translation of the p53 isoform p53/47. Mol Cell 2010;38:78-88.

29. Wang $\mathrm{Y}, \mathrm{Yu} \mathrm{H}$, Zhang J, et al. Hesperidin inhibits HeLa cell proliferation through apoptosis mediated by endoplasmic reticulum stress pathways and cell cycle arrest. BMC Cancer 2015;15:682.

30. Lenna S, Farina AG, Martyanov V, et al. Increased expression of endoplasmic reticulum stress and unfolded protein response genes in peripheral blood mononuclear cells from patients with limited cutaneous systemic sclerosis and pulmonary arterial hypertension. Arthritis Rheum 2013;65:1357-66.

31. Li Z, Zhang L, Wang A, et al. Ectopic overexpression of
SlHsfA3, a heat stress transcription factor from tomato, confers increased thermotolerance and salt hypersensitivity in germination in transgenic Arabidopsis. PLoS One 2013;8:e54880.

32. Lamoureux F, Thomas C, Yin MJ, et al. Suppression of heat shock protein 27 using OGX-427 induces endoplasmic reticulum stress and potentiates heat shock protein 90 inhibitors to delay castrate-resistant prostate cancer. Eur Urol 2014;66:145-55.

33. Park CJ, Seo YS. Heat Shock Proteins: A Review of the Molecular Chaperones for Plant Immunity. Plant Pathol J 2015;31:323-33.

34. Wu J, He GT, Zhang WJ, et al. IRE1alpha Signaling Pathways Involved in Mammalian Cell Fate Determination. Cell Physiol Biochem 2016;38:847-58.

35. Nguyen DT, Kebache S, Fazel A, et al. Nck-dependent activation of extracellular signal-regulated kinase-1 and regulation of cell survival during endoplasmic reticulum stress. Mol Biol Cell 2004;15:4248-60.

36. Zhang LJ, Chen S, Wu P, et al. Inhibition of MEK blocks GRP78 up-regulation and enhances apoptosis induced by ER stress in gastric cancer cells. Cancer Lett 2009;274:40-6.

37. Wang XZ, Ron D. Stress-induced phosphorylation and activation of the transcription factor CHOP (GADD153) by p38 MAP Kinase. Science 1996;272:1347-9.

38. Maytin EV, Ubeda M, Lin JC, et al. Stress-inducible transcription factor $\mathrm{CHOP} /$ gadd153 induces apoptosis in mammalian cells via p38 kinase-dependent and -independent mechanisms. Exp Cell Res 2001;267:193-204.
Cite this article as: Cao L, Yuan X, Bao F, Lv W, He Z, Tang J, Han J, Hu J. Downregulation of HSPA2 inhibits proliferation via ERK1/2 pathway and endoplasmic reticular stress in lung adenocarcinoma. Ann Transl Med 2019;7(20):540. doi: 10.21037/atm.2019.10.16 